\title{
Combined use of urinary Survivin detection and liquid-based cytology for the early diagnosis of bladder urothelial carcinoma
}

\author{
XIAOFENG XU*, PING LI*, DIAN FU, ZHIFENG WEI, SONG XU, FENG XU, \\ FENG TIAN, JINGPING GE, ZHENGYU ZHANG and WEN CHENG
}

Department of Urology, Jinling Hospital, School of Medicine, Nanjing University, Nanjing, Jiangsu 210002, P.R. China

Received October 19, 2015; Accepted January 5, 2018

DOI: $10.3892 / \mathrm{ol} .2018 .8326$

\begin{abstract}
To assess the possibility of combined using urine Survivin and liquid-based cytology (LBC) tests to improve the diagnosis of bladder urothelial carcinoma (BUC), the present study analyzed urine from 36 patients with bladder cancer and 36 benign cases as control individuals. The Survivin content in the patient's morning urine was assessed by ELISA. When the sample value exceeded double of the average of the control group, it was defined as positive and the patient was diagnosed as bladder cancer. Meanwhile, LBC was performed for the same sample. Urinary Survivin detection combined with LBC test showed a sensitivity of $91.7 \%$, a specificity of $91.7 \%$ and accuracy of $91.7 \%$ for the diagnosis of BUC. Compared with each test individually, although the specificity decreased, the sensitivity and accuracy of the combined detection improved significantly $(\mathrm{P}<0.05)$. The data presented in the current study identified that the LBC test is a novel diagnostic method for urinary tract tumors and its detection rate was superior to the conventional urine cytology test. Additionally, Survivin detection combined with LBC could significantly improve the detection rate of BUC. In addition, this method is non-invasive, economical, simple and precise.
\end{abstract}

\section{Introduction}

Bladder cancer $(\mathrm{BCa})$ is the most common cancer of the urinary tract in the United States $(1,2)$. In China, BCa is the malignant tumor of the urinary system with the highest incidence and cancer-associated mortality rate and the incidence of $\mathrm{BCa}$ has

Correspondence to: Professor Wen Cheng or Professor Zhengyu Zhang, Department of Urology, Jinling Hospital, School of Medicine, Nanjing University, 305 East Zhongshan Road, Nanjing, Jiangsu 210002, P.R. China

E-mail: njzychengwen@sina.com

E-mail: njzyzhangzhengyu@sina.com

${ }^{*}$ Contributed equally

Key words: urinary Survivin, liquid-based cytology, combined detection, bladder urothelial carcinoma been increasing in previous years $(3,4)$. Approximately $90 \%$ of $\mathrm{BCa}$ cases are of bladder urothelial carcinoma (BUC) (5). When initially diagnosed, the majority of patients with $\mathrm{BCa}$ undergo transurethral resection of the bladder tumor (TURBt) (6); however, $50-70 \%$ of patients with non-muscle invasive BUC experience disease recurrence following TURBt, 15-25\% of whom experience progression to higher disease grades and stages (7). Early disease diagnosis and surveillance are necessary to attain better outcomes for patients with BUC (8). Currently the standard modalities for detecting $\mathrm{BCa}$ are cystoscopy and urine cytology (8). Cystoscopy is an invasive procedure with high specificity (8). Although urine cytology is non-invasive and has high specificity for detecting carcinoma cells, it lacks sensitivity, particularly for low-grade tumors (8). Therefore, it is necessary to find a non-invasive method with high specificity and sensitivity to improve the diagnosis and surveillance of BUC (9).

Liquid-based cytology (LBC) has been developed as an alternative to conventional urine cytology. Previous comparative studies have shown that $\mathrm{LBC}$ is superior to conventional cytology, as it has a sensitivity and specificity $>90 \%(10-12)$. Additionally, complementary techniques, including DNA, RNA and protein molecular analysis, can be applied to LBC samples, thus allowing the effect of various cancer biomarkers to also be studied $(13,14)$.

In the ongoing search for novel bladder tumor markers, numerous potential markers have been identified; however, their sensitivity and specificity are not high enough to reduce the application of cystoscopies for the detection of bladder carcinoma $(9,15)$. Survivin is a $16.5 \mathrm{kDa}$ protein that acts as a member of the inhibitor of apoptosis proteins (IAP) family and is expressed during embryonic and fetal development (16). Survivin is completely undetectable in normal adult tissue, but is prominently expressed in a variety of the most common human cancer types, including those of the lung, colon, pancreas, prostate and breast $(17,18)$. Owing to its substantial upregulation in cancer tissue and its function in carcinogenesis and tumor progression, Survivin is currently proposed to be a possible tumor marker. In BCa, Survivin expression has been observed by immunohistochemistry and may be associated with accelerated incidence of disease recurrence (19). Survivin has been suggested to be a promising diagnostic marker for $\mathrm{BCa}$ detection owing to its high sensitivity for detection of carcinoma in situ, which is frequently missed by cystoscopy 
and by the majority of other tests (20). Smith et al (21) measured Survivin levels in a group of 138 patients with $\mathrm{BCa}$ and reported a sensitivity of $100 \%$ and a specificity of $95 \%$, where as Wang et al (22) reported a sensitivity of $80 \%$ and a specificity of $100 \%$ in a group of 66 patients with $\mathrm{BCa}$.

At the time of writing, to the best of our knowledge no study has been conducted to investigate the diagnostic efficacy of the combined using of urinary Survivin detection and LBC in the same patients. This preliminary study was therefore undertaken to evaluate the diagnostic values of this combined detection method for BUC.

\section{Patients and methods}

Study subjects. Between January 2012 and December 2013, urine samples from 36 patients with BUC were collected, which were pathologically confirmed following surgery (27 males, 9 females; age range, $41-79$ years; mean age $57.34 \pm 22.20$ years). In total, 26 patients had low-grade (grade I/II) disease and 10 had high-grade (III grade) disease (according to the World Health Organisation 2004 criteria) (23). These patients were divided into 19 cases of non-muscle-invasive tumor (Ta-T1) and 17 cases of muscle-invasive tumor (T2-T4) using the Tumor-Node-Metastasis staging system (UICC 2009) (24). In the control group, 36 patients diagnosed with non-cancerous urinary tract disease were selected (28 males and 8 females, age range, $40-78$ years; 13 cases of benign prostate hyperplasia, 6 of chronic prostatitis, 8 of urinary tract stones, 3 of urinary tract infection, 4 of glandular cystitis, 1 of redundant prepuce and 1 of foreign body of bladder). Fasting morning urine samples were obtained from all subjects. The present study was approved by the ethics committee of Jinling Hospital and each patient provided written informed consent.

Sample collection. Midstream urine samples $(40-50 \mathrm{ml})$ were obtained from all subjects and were concentrated immediately by centrifugation at $400 \mathrm{xg}$ for $5 \mathrm{~min}$ at room temperature $\left(22 \pm 1^{\circ} \mathrm{C}\right)$. The samples were then stored at $-80^{\circ} \mathrm{C}$ until further processing.

ELISA. Survivin protein levels were measured in urine with Human Survivin ELISA kit (D10564; Jingmei Biotech Co., Ltd., Shenzhen, China) according to the manufacturers protocol. The absorbance was measured using a microplate reader at $570 \mathrm{~nm}$ (Model 680 Microplate Reader; Bio-Rad Laboratories, Inc., Hercules, CA, USA) and the data were recorded. A Survivin concentration that was 2-fold higher than the mean levels of control groups were defined as positive, as previously described (19).

$L B C$ test. The LBC test was performed using an automated liquid-based monolayer cell preparation system (ThinPrep 2000 system; Hologic, Inc., Marlborough, MA, USA). The samples were immersed in Cytolyt (Hologic, Inc., Marlborough, MA, USA) and transferred to a PreservCyt bowl. The cylinder with a filtration membrane was then placed in PreservCyt bowl and was rotated to ensure that the cells were homogeneously distributed. When a vacuum was applied, the erythrocytes and mucus were removed by negative pressure. Thus, only the cells left on the filtration membrane were attached to the slide and then fixed in 95\% ethyl alcohol at room temperature. Following fixation for $30 \mathrm{~min}$, smears of specimens were cytologically stained using standard Papanicolaou staining as previously described (25). All slides were evaluated routinely by an experienced cytopathologist without any prior knowledge of the immunohistochemical findings.

Statistical analysis. The results are presented as proportion (\%). The sensitivity, specificity and accuracy were calculated for different detection method. Comparison of the results were performed by the $\chi^{2}$ test (SPSS 11.0 for Windows; SPSS, Inc., Chicago, IL, USA). P $<0.05$ was considered to indicate a statistically significant difference.

\section{Results}

Survivin level is elevated in the urine of patients with BUC. The clinical utility of Survivin at discriminating patients with BUC from individuals with non-cancerous urinary tract disease was analyzed. Urine samples were categorized according to histopathological results. Survivin concentrations in individual urine samples were measured using a commercial ELISA kit. Survivin levels in the urine of BUC and non-cancerous urinary tract disease are demonstrated in Table I. Of the 36 BUC cases, 21 were Survivin-positive. In the non-cancerous urinary tract disease group, only 2 cases were Survivin-positive. The results revealed that urine Survivin concentrations are extremely high in the BUC group compared with the controls. The presence of Survivin was able to discriminate bladder cancer from benign disease.

Most of BUC cases test positive by LBC. The LBC diagnoses for the 72 cytological samples are summarized in Table I. The cells were uniformly distributed on the glass slides in all cases. The LBC technique provides a clean background with less obscuring inflammatory cells and blood compared with urine cytology (Fig. 1). Of the 36 cancer cases, 28 were found to be positive by LBC; of the 36 cases in control group, 1 was suspected to be positive.

The combined use of Survivin detection and LBC has a significantly increased diagnostic sensitivity and accuracy. The results for the combined use of Survivin detection and LBC had also been indicated in the Table I. Of 36 cases in BUC group, 33 were Survivin- and LBC-positive. Compared with Survivin detection or LBC alone, the combined detection method had a slightly lower specificity, but a significantly increased diagnostic sensitivity and accuracy $(\mathrm{P}<0.05)$.

\section{Discussion}

An ideal test to monitor BCa should be objective, non-invasive, easy to administer and interpret, and have high sensitivity and specificity. An ideal tumor biomarker should be highly sensitive, specific and accurate (26). Ease of performance and low cost are also desirable. It has been reported that Survivin and LBC have utility for the detection of BUC $(11,12,16,20,22,26-36)$.

Urinary levels of Survivin expression at the protein and mRNA levels are associated with BCa presence, higher 
Table I. Combined sensitivity, specificity and accuracy for Survivin and LBC in detection of bladder cancer.

\begin{tabular}{lccc}
\hline Parameter(s) & Sensitivity, \% (n) & Specificity, \% (n) & Accuracy, \% (n) \\
\hline Survivin & $58.0(21 / 36)^{\mathrm{a}}$ & $94.4(34 / 36)$ & $76.4(55 / 72)^{\mathrm{a}}$ \\
LBC & $77.8(28 / 36)^{\mathrm{a}}$ & $97.2(35 / 36)$ & $87.5(63 / 72)^{\mathrm{a}}$ \\
Survivin and LBC & $91.7(33 / 36)^{\mathrm{a}}$ & $91.7(33 / 36)$ & $91.7(66 / 72)^{\mathrm{a}}$ \\
\hline
\end{tabular}

LBC, liquid-based cytology; ${ }^{\mathrm{P}}<0.05$.
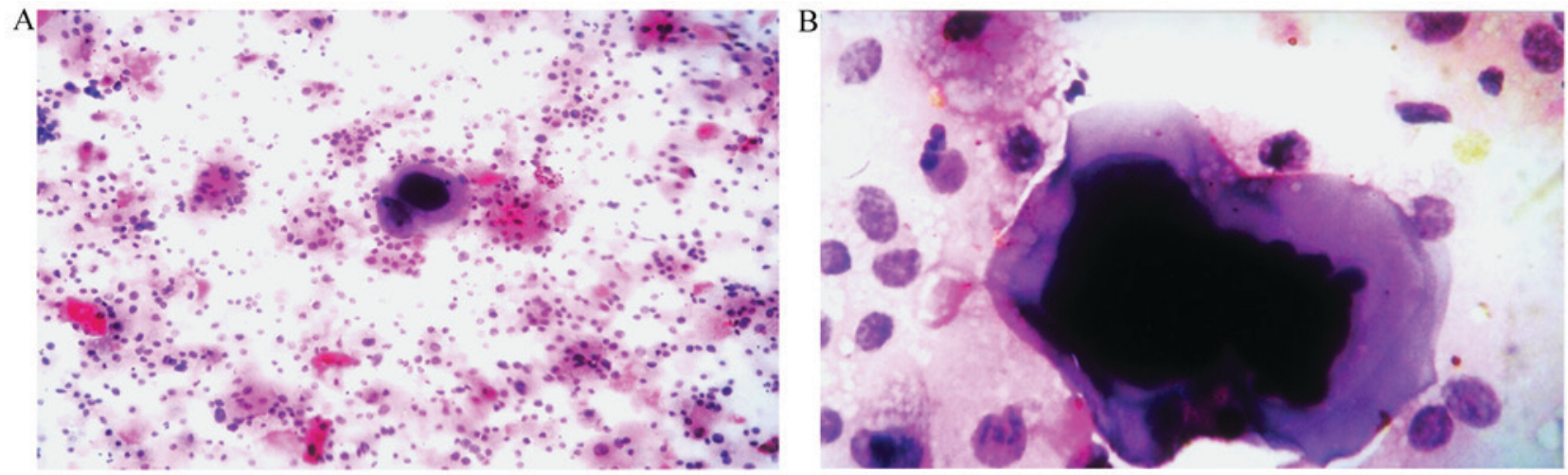

Figure 1. Representative examples of liquid-based cytology. (A) Bladder urothelial carcinoma cells (magnification, x200). (B) Single giant malignant cells with hyperchromatic nuclei and irregular nuclear membranes (magnification, x400).

tumor grade, and advanced pathologic stage (16-28). Survivin protein levels have been detected previously by nested polymerase chain reaction (nested PCR), immunohistochemical staining, western blot analysis and ELISA, with the nested PCR most widely used $(21,29,30)$. Smith et al (21) were the first to evaluate the diagnostic potential of Survivin expression in bladder cancer, using protein and mRNA detection methods. The sensitivity of Survivin as a biomarker was $100 \%$, with a specificity of $95 \%$ in a group of 138 patients (21). Zhao et al (29) reported that molecular beacons detected cancerous cells in $28(80 \%)$ of the 35 patients with confirmed $\mathrm{BCa}$. The Survivin mRNA assay for the diagnosis of $\mathrm{BCa}$ had only $68.6 \%$ sensitivity; however, it did have $100 \%$ specificity. Chen et al (30) found that Immunohistochemical analysis indicated high survivin expression was associated with tumor stage and grade and may present a predictive marker of overall survival in BUC. Zhao et al (29) reported that Survivin protein expression was detected in $25(71.4 \%)$ of the 35 patients studied. The sensitivity and specificity of ELISA was 54.3 and $68.6 \%$, respectively. In summary, Survivin has been shown to be implicated in the detection of BUC $(21,29,30)$. The findings indicate that Survivin is a promising urinary molecular marker for BCa early detection and prognosis prediction $(21,29,30)$. However, the assays above are non-standardized, as none of their performances complied with validation criteria appropriate for analytic techniques and the assays require further innovation and standardization. Eissa et al (19) reported that qualitative reverse transcription-PCR in 153 samples revealed that the mean rank levels of survivin in the benign and malignant groups were significantly increased by 1.1 and 2.23 -fold, respectively, vs. the normal group $(\mathrm{P}<0.0001)$. In the present study, Survivin levels over 2 times of mean value in control group for the sample were defined as positive. Applying this cutoff value, the sensitivity and specificity of urinary survivin was 58.0 and $94.4 \%$, respectively.

Urine cytology, as a diagnosis method, has a reasonable sensitivity for the detection of high-grade urothelial carcinoma (UC); however, it is less sensitive for detecting low-grade tumors (31).

LBC have been regarded as an alternative to conventional cytology for the primary screening of BCa owing to its practical advantages, with variable circumstances. The advantages of LBC include: samples have a 'cleaner' background, devoid of blood, mucus and inflammatory cells; LBC uses automated and standardized processing techniques that produces the homogenous distribution and efficient fixation of cells; and the residual biological material can be used for immunohistochemical or other special analyses (32-34). Several previous reports have demonstrated that $\mathrm{LBC}$ is superior to conventional cytology for the identification of $\mathrm{BCa}(11,35,36)$. LBC has a sensitivity and specificity $>90 \%$ in non-gynecological specimens (12). The present study revealed LBC had a sensitivity of $77.8 \%$ and specificity of $97.2 \%$.

To the best of our knowledge, the present study is the first to investigate the diagnostic activity of Survivin and LBC in the same patients with BUC. The results indicated that combined use of urine Survivin and LBC test could significantly improve the diagnosis of BUC. In the present study, the efficacy of this combined test was investigated, which exhibited a sensitivity of $91.7 \%$, specificity of $91.7 \%$ and accuracy of $91.7 \%$. Compared with the results of the individual tests, the specificity was decreased, but the sensitivity and accuracy improved significantly. More importantly, this method 
is non-invasive, economical, simple and precise. Therefore, in future LBC could be used in combination with Survivin detection for more accurate results in the diagnosis of BUC. The research discussed in the current study is limited owing to the small number of samples. Further investigation is therefore required, with a study involving a large number of patients.

\section{Acknowledgements}

Not applicable.

\section{Funding}

This work was supported by the grants from the National Natural Science Foundation of China (No. 81572526; 30901716).

\section{Availability of data and materials}

The datasets used and analyzed during the current study are available from the corresponding author on reasonable request.

\section{Authors' contributions}

WC had full access to all the data in the study and takes responsibility for the integrity of the data and the accuracy of the data analysis. Study concept and design: WC. Acquisition of data: WC, XX, PL, DF, ZW and SX. Analysis and interpretation of data: WC, XX, PL, ZYZ and JG. Drafting of the manuscript: $X X$. Critical revision of the manuscript for important intellectual content: WC, XX and PL. Statistical analysis: FX, FT. Supervision: ZYZ and JG.

\section{Ethics approval and consent to participate}

The present study was approved by the Ethics Committee of Jinling Hospital (Nanjing, China) and all patients provided written informed consent.

\section{Consent for publication}

All patients provided written informed consent for publication.

\section{Competing interests}

The authors declare that they have no competing interests.

\section{References}

1. Siegel R, Ma J, Zou Z and Jemal A: Cancer statistics, 2014. CA Cancer J Clin 64: 9-29, 2014

2. Jacobs BL, Lee CT and Montie JE: Bladder cancer in 2010: How far have we come? CA Cancer J Clin 60: 244-272, 2010.

3. Parkin MD, Bray F, Ferlay J and Pisani P: Global cancer statistics, 2002. CA Cancer J Clin 55: 74-108, 2005.

4. Sujun H, Siwei Z, Wanqing C and Chang-Ling L: Analysis of the status and trends of bladder cancer incidence in China. Oncol Prog 11: 89-95, 2013.

5. Fleshner NE, Herr HW, Stewart AK, Murphy GP, Mettlin C and Menck HR: The national cancer data base report on bladder carcinoma. The American college of surgeons commission on cancer and the american cancer society. Cancer 78: 1505-1513, 1996.

6. Richterstetter M, Wullich B, Amann K, Haeberle L Engehausen DG, Goebell PJ and Krause FS: The value of extended transurethral resection of bladder tumour (TURBT) in the treatment of bladder cancer. BJU Int 110: E76-E79, 2012 .
7. Brausi M,Collette L, Kurth K, van der Meijden AP, Oosterlinck W, Witjes JA, Newling D, Bouffioux C and Sylvester RJ; EORTC Genito-Urinary Tract Cancer Collaborative Group: Variability in the recurrence rate at first follow-up cystoscopy after TUR in stage Ta T1 transitional cell carcinoma of the bladder: A combined analysis of seven EORTC studies. Eur Urol 41: 523-531, 2002.

8. Schmitz-Dräger BJ, Droller M, Lokeshwar VB, Lotan Y, Hudson MA, van Rhijn BW, Marberger MJ, Fradet Y, Hemstreet GP, Malmstrom PU, et al: Molecular markers for bladder cancer screening, early diagnosis and surveillance: The WHO/ICUD consensus. Urol Int 94: 1-24, 2015.

9. Goebell PJ, Groshen SL and Schmitz-Dräger BJ: Guidelines for development of diagnostic markers in bladder cancer. World $\mathbf{J}$ Urol 26: 5-11, 2008.

10. Nicol TL, Kelly D, Reynolds L and Rosenthal DL: Comparison of TriPath thin-layer technology with conventional methods on non-gynecologic specimens. Acta Cytol 444: 567-575, 2000.

11. Bishop JW, Bigner SH, Colgan TJ, Husain M, Howell LP, McIntosh KM, Taylor DA and Sadeghi MH: Multicenter masked evaluation of AutoCyte PREP thin layers with matched conventional smears. Including initial biopsy results. Acta Cytol 42: 189-197, 1998.

12. Nasuti JF, Tam D and Gupta PK: Diagnostic value of liquid based (ThinPrep) preparation in non-gynecologic cases. Diagn Cytopathol 24: 137-141, 2001

13. Tisserand P, Fouquet C, Marck V, Mallard C, Fabre M, Vielh P and Soussi T: Thinprep-processed fine-needle samples of breast are effective material for RNA- and DNA-based molecular diagnosis. Application to p53 mutation analysis. Cancer 99: 223-232, 2003.

14. Linder J: Recent advances in thin-layer cytology. Diagn Cytopathol 18: 24-32, 1998.

15. Weikert S, Christoph F, Schrader M, Krause H, Miller K and Müller M: Quantitative analysis of survivin mRNA expres-sion in urine and tumour tissue of bladder cancer patients and its potential relevance for disease detection and prognosis. Int J Cancer 116: 100-104, 2005.

16. Horstmann M, Bontrup H, Hennenlotter J, Taeger D, Weber A, Pesch B, Feil G, Patschan O, Johnen G, Stenzl A and Brüning T: Clinical experience with survivin as a biomarker for urothelial bladder cancer. World J Urol 28: 399-404, 2010.

17. Adida C, Crotty PL, McGrath J, Berrebi D, Diebold J and Altieri DC: Developmentally regulated expression of the novel cancer anti-apoptosis gene survivin in human and mouse differentiation. Am J Pathol 152: 43-49, 1998.

18. Tanaka K, Iwamoto S, Gon G, Nohara T, Iwamoto M and Tanigawa N: Expression of survivin and its relationship to loss of apoptosis in breast carcinomas. Clin Cancer Res 6: 127-134, 2000.

19. Eissa S, Swellam M, Shehata H, El-Khouly IM, El-Zayat T and El-Ahmady O: Expression of HYAL1 and survivin RNA as diagnostic molecular markers for bladder cancer. J Urol 183: 493-498, 2010.

20. Ku JH, Godoy G, Amiel GE and Lerner SP: Urine survivin as a diagnostic biomarker for bladder cancer: A systematic review. BJU Int 110: 630-636, 2012.

21. Smith SD, Wheeler MA, Plescia J, Colberg JW, Weiss RM and Altieri DC: Urine detection of survivin and diagnosis of bladder cancer. JAMA 285: 324-328, 2001.

22. Wang H, Xi X, Kong X, Huang G and Ge G: The expression and significance of survivin mRNA in urinary bladder carcinomas. J Cancer Res Clin Oncol 130: 487-490, 2004.

23. Eble JN, Sauter G, Epstein JI and Sesterhenn IA (eds.): World Health Organization Classification of Tumours. Pathology and Genetics of Tumours of the Urinary System and Male Genital Organs. IARC Press, Lyon, 2004.

24. Sobin LH, Gospodariwicz M and Wittekind C; International Union against Cancer: TNM classification of malignant tumours. 2009, UICC international union against cancer. 7th edition. Wiley-Blackwell, Hoboken, NJ, pp262-265, 2009.

25. Gurley AM, Hidvegi DF, Bacus JW and Bacus SS: Comparison of the papanicolaou and feulgen staining methods for DNA quantification by image analysis. Cytometry 11: 468-474, 1990.

26. Goodison S, Rosser CJ and Urquidi V: Bladder cancer detection and monitoring: Assessment of urine- and blood-based marker tests. Mol Diagn Ther 17: 71-84, 2013.

27. Pina-Cabral L, Santos L, Mesquita B, Amaro T, Magalhães S and Criado B: Detection of survivin mRNA in urine of patients with superficial urothelial cell carcinomas. Clin Transl Oncol 9: 731-736, 2007. 
28. Srivastava AK, Singh PK, Srivastava K, Singh D, Dalela D, Rath SK, Goel MM and Brahma Bhatt ML: Diagnostic role of survivin in urinary bladder cancer. Asian Pac J Cancer Prev 14: 81-85, 2013.

29. Zhao J, Wang ZQ, Wang XY, Yang XJ and He D: Preliminary study of diagnostic utility of molecular beacons in bladder cancer. Urology 76: 512.e8-e13, 2010.

30. Chen HA, Su CM, Hsieh HY, Tung CL, Hsu CD, Wang YH and Shen CH: Clinical significance of survivin expression in patients with urothelial carcinoma. Dis Markers 2014: 574985, 2014.

31. Wiener HG, Mian C, Hiatel A, Pycha A, Schatzl G and Marberger M: Can urine bound diagnostic tests replace cystoscopy in the management of bladder cancer? J Urol 159: 1876-1880, 1998.

32. Lu DY, Nassar A and Siddiqui MT: High-grade urothelial carcinoma: Comparison of SurePath liquid-based processing with cytospin processing. Diagn Cytopathol 37: 16-20, 2009.

33. Koo JH, Lee HC, Song HG, Han HS, Lee KH, Choe KH, Lee KM and Lee OJ: Comparison of cytologic evaluation between conventional method and Cellprep PLUS liquid-based cytology in body fluid. Korean J Pathol 43: 448-452, 2009.
34. Son SM, Koo JH, Choi SY, Lee HC, Lee YM, Song HG, Hwang HK, Han HS, Yun SJ, Kim WJ, et al: Evaluation of urine cytology in urothelial carcinoma patients: A comparison of cellprepPlus ${ }^{\circledast}$ Liquid-Based cytology and conventional smear. Korean J Pathol 46: 68-74, 2012.

35. Nicol TL, Kelly D, Reynolds L and Rosenthal DL: Comparison of TriPath thin-layer technology with conventional methods on non-gynecologic specimens. Acta Cytol 44: 567-575, 2000.

36. Fischler DF and Toddy SM: Nongynecologic cytology utilizing the Thin-Prep Processor. Acta Cytol 40: 669-675, 1996.

(c) (i) () $($ This work is licensed under a Creative Commons cc) Attribution-NonCommercial-NoDerivatives 4.0 International (CC BY-NC-ND 4.0) License. 